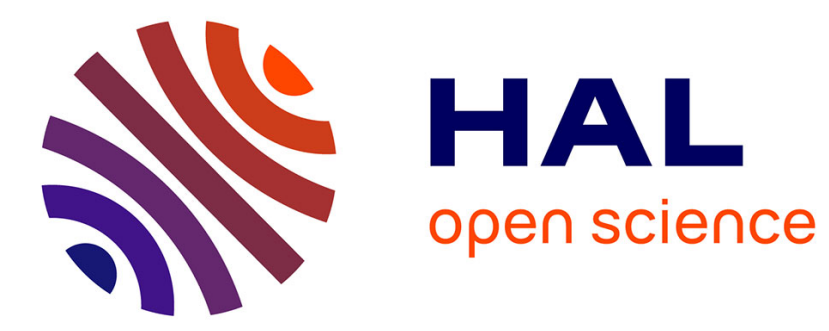

\title{
Framework for a distributed and hybrid prognostic system.
}

Otilia Elena Dragomir, Rafael Gouriveau, Noureddine Zerhouni, Florin

Dragomir

\section{- To cite this version:}

Otilia Elena Dragomir, Rafael Gouriveau, Noureddine Zerhouni, Florin Dragomir. Framework for a distributed and hybrid prognostic system.. 4th IFAC Conference on Management and Control of Production and Logistics, MCPL'2007., Sep 2007, Sibiu, Romania. pp.431-436. hal-00181342

\section{HAL Id: hal-00181342 \\ https://hal.science/hal-00181342}

Submitted on 23 Oct 2007

HAL is a multi-disciplinary open access archive for the deposit and dissemination of scientific research documents, whether they are published or not. The documents may come from teaching and research institutions in France or abroad, or from public or private research centers.
L'archive ouverte pluridisciplinaire HAL, est destinée au dépôt et à la diffusion de documents scientifiques de niveau recherche, publiés ou non, émanant des établissements d'enseignement et de recherche français ou étrangers, des laboratoires publics ou privés. 


\title{
FRAMEWORK FOR A DISTRIBUTED AND HYBRID PROGNOSTIC SYSTEM
}

\author{
Otilia Elena DRAGOMIR ${ }^{1}$, Rafael GOURIVEAU ${ }^{2}$, Noureddine ZERHOUNI ${ }^{3}$, Florin DRAGOMIR ${ }^{4}$
}

\author{
1,2,3 Laboratoire d'Automatique de Besançon (LAB) \\ 24 Rue Alain Savary, 25000 Besançon, France \\ tel/fax: +33 (0)381 402801 /28 09, e-mai :drg_otilia@yahoo.com, \\ \{rgourive,zerhouni\}@ens2m.fr \\ 4 Valahia University of Târgovişte, \\ Electrical Engineering Faculty, Automation and Information Department \\ Unirii Avenue 18-20, Târgoviște, România \\ telffax0245217683,e-mail:\{drg_florin\}@yahoo.com
}

\begin{abstract}
The purpose of this paper is to define a framework for the implantation of a distributed, adaptable and open prognostic system able to take into account, on one hand, the dynamic of the monitored equipment and, on the other hand, the evolution of performance criteria. In this way, the prognostic process is (re)defined: at the component level and at the global level of the system (whole equipment). In the distributed model proposed, the interest of neural networks as "prognostic tools" is pointed out. The work is in coherence with actual industrial maintenance developments like "e-maintenance systems" or "web-services applications". Copyright @ 2007 IFAC
\end{abstract}

Keywords: maintenance, degradation, performance, monitoring, prediction, distributed, neural networks

\section{INTRODUCTION}

The maintenance activity combines different methods, tools and techniques to reduce maintenance costs while increasing reliability, availability and security of equipments. Thus, one usually speaks about fault detection, failures diagnostic, response development (choice of management actions preventive and/or corrective) and scheduling of these actions. Briefly these steps correspond to the need, firstly, of "perceiving" phenomena, next, of "understanding" them, and finally, of "acting" consequently. However, rather than understanding a phenomenon which has just appeared like a failure (a posteriori comprehension), it seems convenient to "anticipate" it's manifestation in order to consequently and, as soon as possible, resort to protective actions. This is what could be defined as the "prognostic process" and which the object of this paper is.
Prognostic reveals to be a very promising maintenance activity as it should permit to not engage inopportune maintenance spending. Also, industrials show a growing interest in this thematic which becomes a major research framework; see recent papers dedicated to "CBM", condition-based maintenance (Jardine, et al., 2006; Ciarapica and Giacchetta, 2006). The relative positioning of detection, diagnostic, prognostic and decision / scheduling can be schematized as proposed in Fig. 1.

The research community still doesn't propose a formal framework to instrument the prognostic process. (1) The prognostic itself isn't a stabilized concept. (2) Existing developments deal essentially with the provisional assessment of component's states and leave side a "system-approach" of the problem. (3) This is all the more critical as systems evolve in complexity (mecatronics systems) and in scale (distributed systems)... 


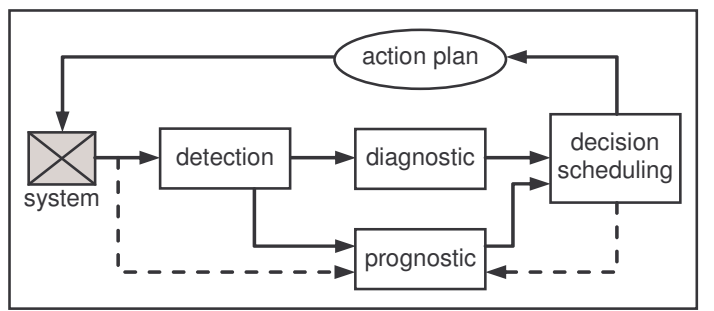

Fig. 1. Relative positioning of detection, diagnostic, prognostic and decision/scheduling activities.

In this context, the purpose of the work reported here is to define a framework for the implantation of a distributed prognostic system to ensure a predictive analysis at the global level of the system (the whole equipment) and enable to take into account, on one hand, the dynamic of the monitored equipment and, on the other hand, the evolution of performance criteria.

The paper is organized in three parts. First of all (section 2), the concept of "prognostic" is briefly defined and positioned within the maintenance strategies. Some developments have been led to improve the proactive capacities of maintainers. So, the next part is dedicated to the analysis of the tools used in time forecasting (section 3). At this stage, a special attention is given to neural networks which main advantages in regards with the reported work are pointed out. Finally (section 4), an hybrid and distributed system for "prognostic instrumentation" is proposed. The whole aims at bringing the analysis from local (component) to global (system) level.

\section{MAINTENANCE AND PROGNOSTIC}

\subsection{Prognostic as a key feature of maintenance policies}

As mentioned earlier, the growth of the reliability, availability or safety of a system is a determining factor in regard with the effectiveness of industrial maintenance. As a consequence, the implementation of provisional strategies is a good way to improve the availability of processes, to ensure the smallest variations of products qualities or the direct costs falling (Leger and Morel, 2001). Furthermore, durable development involves the integration of economical strategies beside social and environmental ones for the optimization of processes. This major provocation of triple performance outlined a new and interesting research area in scientific world: concepts like statical corrective or preventive maintenance were replaced by reactive or proactive maintenance (Iung, et al. 2003) and the prognostic take a growing place. Obviously, considering the benefits that a "prognostic system" may bring to the security, economics and resource management fields, the industrial community takes now interest in this area. In a word "prognostic" is recognized as a key feature in maintenance strategies. Let's have a closer look on this concept.

\subsection{Acceptations of "prognostic"}

The European Standard on maintenance terminology (EN 13306, 2001) doesn't define the "prognostic". It doesn't appear either on the IFAC keyword list. This reveals that there is no consensual acceptation of this term. The literature on prognostics is still small. The objective of a prognostic "module" can be summarized as follows.

Historical acceptation. Prognosis is traditionally related to fracture mechanics and fatigue. It started to be brought up by the modal analysis community as a field of interest (Farrar, et al., 2003). In this "meaning", prognostic is also called the prediction of a system's lifetime, which corresponds to the last level of the classification of damage detection methods introduced by (Rytter, 1993). This acceptation of "prognostic" can be generalized by the RUL concept (see here after).

Prognostic as a RUL estimation. The most widely definition of prognostic is the following: a process whose objective is to predict how much time is left before a failure (or a fault) occurs given the current machine condition and past operation profile (Jardine, et al., 2006). The time left before observing a failure is usually called the "remaining useful life" (RUL), remaining service life, residual life, or remnant life.

Prognostic as a probability measure. Prognostic can aim at predicting the chance that a machine operates without a fault or failure up to some future time. In the general maintenance context, this "probabilistic prognostic value" is all the more an interesting indication as the fault or failure can have catastrophic consequences (e.g. nuclear power plant): maintenance manager need to know if the inspection interval is appropriate... However, a small number of papers address this second type of prognostics (Lin and Makis, 2003; Farrar, et al., 2003).

\section{3 "Prognostic" as an assessment process}

Predict. All definitions proposed here above assimilate prognostic to a "prediction process": a future situation must be caught. In addition, this obviously supposes that the current situation can be grasped (practically, it's the synthesis of a detection process and of measured data of the system).

Assess. More over, these approaches are grounded on the failure (or fault) notion, which implies that the "prognostic activity" is associated with a degree of acceptability (a system must perform a required function: EN 13306, 2001). Thus, prognostic should be based on assessment criteria, whose limits depend on the system itself and on performance objectives.

Consider Fig. 2 to illustrate this assumption: the predicted situation at time " $\mathrm{t}+\mathrm{dt}$ " can be considered as a critical one because of the degradation limit. Without this limit, there is no way to conclude on the predicted situation. 


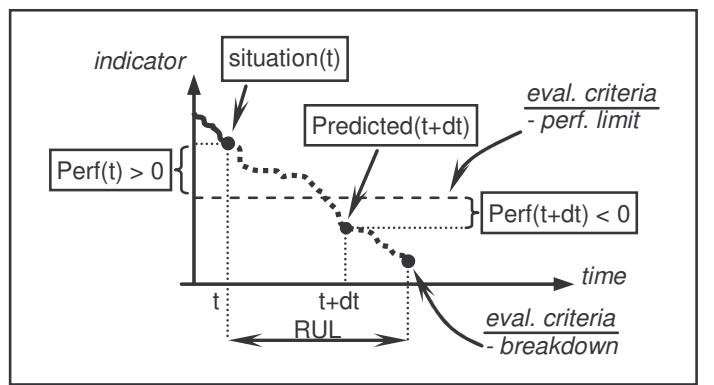

Fig. 2. Simple illustration of the difference between prediction and performance evaluation.

Thus, prognostic could be split into 2 sub-activities: a first one to predict the evolution of a situation at a given time, and a second one to assess this predicted situation with regards to an evaluation referential. Let's resume (Fig. 3.):

- identification: a situation is captured by the detection process and additional current measures,

- prediction: the situation is forecasted in time,

- assessment: a situation is evaluated by the use of performance criteria,

- prognostic: a predicted situation is assessed.

The difference pointed out here between prediction and prognostic can be assimilated to this one between "information" and "knowledge" concepts: the prediction sub-process informs on a phenomenon (from data to information), and the assessment one's enable to interpret it and pro-act on it (from information to knowledge). Obviously, in practical situation all this must be supported by operational tools. This is the purpose of section 3 .

\section{PROGNOSTIC METHODS}

\subsection{Overview}

Various approaches to prognostics have been developed that range in fidelity from simple historical failure rate models to high-fidelity physicsbased models (Byington, 2002).

The required information (depending on the type of prognostics approach) include: engineering model and data, failure history, past operating conditions, current conditions, identified fault patterns, transitional failure trajectories, maintenance history, system degradation and failure modes.

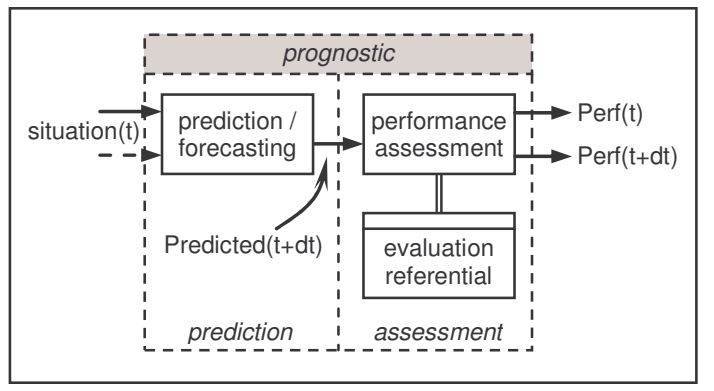

Fig. 3. Prognostic as a prediction and assessment process.
Let's have an overview of examples of prognostics approaches that have successfully been applied for different types of problems.

- Experience-Based Prognostics. Use statistical reliability to predict probability of failure at any point in time.

- Evolutionary/Statistical Trending Prognostics. Multi-variable analysis of system response and error patterns compared to known fault patterns.

- Artificial Intelligence Based Prognostics. Mechanical failure prediction using reasoners trained with failure data.

- State Estimator Prognostics. System degradation or diagnostic feature tracking using Kalman filters and other predictor-corrector schemes.

- Model-Based or Physics of Failure Based Prognostics. Fully developed functional and physics-of-failure models to predict degradation rates given loads and conditions.

Similar to diagnosis, prognostic methods can be classified as being associated with one of the following two approaches: model-based and datadriven (Chiang, et al., 2001). Each one of these approaches has its own advantages and disadvantages, and, consequently, they are often used in combination in many applications. The next paragraphs present a synthesis of it.

Let's note that according to some authors, all methods are labeled as "prognostic tools". However, most of them refer to what, in this paper, is called "prediction"...

\subsection{Model based approaches}

The model-based methods assume that an accurate mathematical model can be constructed from first principles. As an example, physics-based fatigue models have been extensively employed to represent the initiation and propagation of structural anomalies. The model-based methods often use residuals as features, where the residuals are the outcomes of consistency checks between the sensed measurements of a real system and the outputs of a mathematical model. The premise is that the residuals are large in the presence of malfunctions, and small in the presence of normal disturbances, noise and modeling errors.

Statistical techniques are used to define thresholds to detect the presence of faults. The three main ways of generating the residuals are based on:

- parameter estimation,

- observers (Kalman filters, reduced order unknown input observers, Interacting Multiple Models),

- parity relations.

Advantage and drawback. The main advantage of model-based approaches is their ability to incorporate physical understanding of the system to be monitored. In addition, in many situations, the changes in feature vector are closely related to model parameters (Chelidze, et al., 2002). 
Therefore, model based approaches can also establish a functional mapping between the drifting parameters and the selected prognostic features. Moreover, if the understanding of the system degradation improves, the model can be adapted to increase its accuracy and to address subtle performance problems. Consequently, it can significantly outperform data-driven approaches. But this closed relation with a mathematical model may also be a strong weakness: it can be difficult even impossible to catch the system behavior (distributed systems, mecatronics systems,...). Further, some authors think that the monitoring and prognostic tools must evolve as the system does...

\subsection{Data driven approaches}

Data-driven approaches derive directly from routinely monitored system operating data (e.g., calorimetric or spectrometric data, power, vibration and acoustic signal, temperature, pressure, oil debris, currents voltages...). In many applications, measured input/output data is the major source for a deeper understanding of the system degradation behavior.

Data-driven approaches rely on the assumption that the statistical characteristics of data are relatively unchanged unless a malfunctioning even occurs. According to the scientific literature, data-driven approaches can be divided on two global categories:

- statistical techniques:

- multivariate statistical methods (static and dynamic principle components (PCA),

- linear and quadratic discriminant,

- partial least squares (PLS),

- canonical variates analysis (CVA),

- signal analysis (niters, auto-regressive models, FFT, etc.).

- artificial intelligent techniques:

- neural networks (multi-layer perceptron, probabilistic neural networks, learning vector quantization, self-organizing maps, etc.),

- fuzzy rule-based systems,

- decision trees,

- graphical models (Bayesian networks, hidden Markov models).

Case-based Reasoning (CBR), intelligent decisionbased models and min-max graphs have been considered as potential candidates for prognostic algorithms too.

Advantage and drawback. The strength of datadriven techniques is their ability to transform highdimensional noisy data into lower dimensional information for diagnostic/prognostic decisions. AI techniques have been increasingly applied to machine prognostic and have shown improved performances over conventional approaches.

In practice however, it isn't easy to apply AI techniques due to the lack of efficient procedures to obtain training data and specific knowledge. So far, most of the applications in the literature just use experimental data for model training. Thus, datadriven approaches are highly-dependent on the quantity and quality of system operational data.

\subsection{Artificial Neural Networks}

Within the field of maintenance problems, Artificial Neural Networks (ANNs) have successfully been used to support the detection, diagnostic and prediction processes, and research works emphasize on the interest of using it (Dong, et al., 2004; Freitas, et al., 1999; Wang and Vachtsevanos, 2001; Yam, et al., 2001; Zhang, et al., 1998): ANNs are a general and flexible modeling tool, especially for prediction problems. Let's point out the principle arguments of this assumption (not exhaustive list).

An adaptable tool. ANNs are data-driven selfadaptive methods in that they learn from examples and capture subtle functional relationships among the data, even if the underlying relationships are unknown. Thus, ANNs are well suited for problems whose solutions require knowledge that is difficult to specify but for which there are enough data or observations.

A robust tool. After the learning phase, ANNs can often correctly infer the unseen part of a population even if the sample data contain noisy information.

A general tool. ANNs are capable of performing nonlinear modeling which is a really interesting characteristic as many real world systems are nonlinear too.

An open tool. In recent works, extensions of ANNs like neuro-fuzzy systems have been developed in order to overpass the performance of classical neural networks, in particular for prediction problems. See (Wang, et al. 2004), or (Ciarapica and Giacchetta, 2006) for an example.

\section{TOWARDS AN HYBRID AND DSTRIBUTED PROGNOSTIC SYSTEM}

In this part, to avoid misunderstandings, the terms "system" and "tool" are distinguished: "system" refers to the equipment to be monitored and "tool" designates the way of supporting the "prognostic process" (implementation of a method or technique).

\subsection{Prognostic tool requirements: the global point of view}

A few requirements of general prognostic tools emerge from sections 2 and 3 .

First of all, a prognostic tool must obviously enable the prediction of a future state of the system. In addition, managers do not only want to know what a situation will be, but evaluate this one too: which performance will be reached? However, each component of the system can be considered as a single system which involves that there is no general way of defining performances. Moreover, actual real systems can't be considered as static and definitive systems... Thus, the prognostic tools must be dynamic and adaptable to be suitable. 


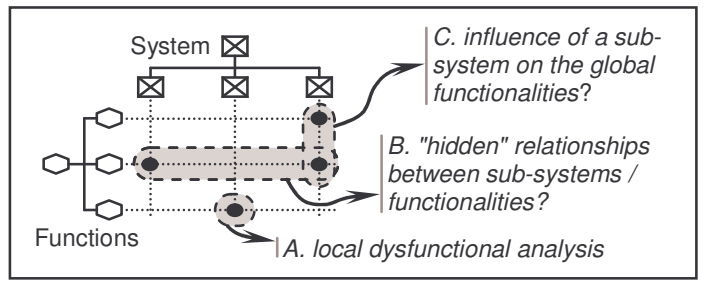

Fig. 4. Relationships between system structure and functions in a dysfunctional analysis.

\subsection{Prognostic tool requirements: functionalities to be provided}

Generally, a system analysis is performed by exploiting different models: a functional model to describe the functions to be provided by the system, a structural model to describe the architecture allowing the realization of these functions, and an event model to describe the behavior. Diverse requirements for a general prognostic tool follow from it. Consider Fig. 4 to argue.

The system as a sum of components - local prognostics (point $A$ in Fig. 4). Considering subsystems, one must be able to "locally" predict the comportment of the component and to assess the performances reached. Now, this kind of prognostic is all the more reliable as the local behavior of the system and the local expectations from it are considered, which implies the development of specifics but accurate prognostic tools.

The system as a set-global prognostic (points B and $C$ in Fig. 4). From the health-management point of view, relationship between function, structure and behavior can be difficult to identify (complexity of the process, lack of knowledge, complicated causal relations, etc.). These considerations make harder the modeling step, even impossible. Yet, a monitoring process must deal with it, and artificial intelligence should be valorized to support a global prognostic. (Note that the same reasons led researchers to associate dependability tools like "fault trees" with artificial intelligence in diagnostic applications.)

\subsection{An hybrid and distributed prognostic tool}

According to all considerations made overhead, the evolution and the complexity of actual systems can not be handled for all characteristics: the analyses are made at the component level and the global aspect of the system isn't considered in classical approaches. Moreover, a prognostic tool has to dynamically adapt itself to follow the system monitored evolution. Thus, a framework for prognostic should be the integration of various specifics prognostic tools by a generic one called "intelligent integrator" (Fig. 5).

Specific tools. There is no preferential tool to perform local prognostics: each one must be the most appropriated according to the component monitored. However, "local performance assessment" should be handled by them.

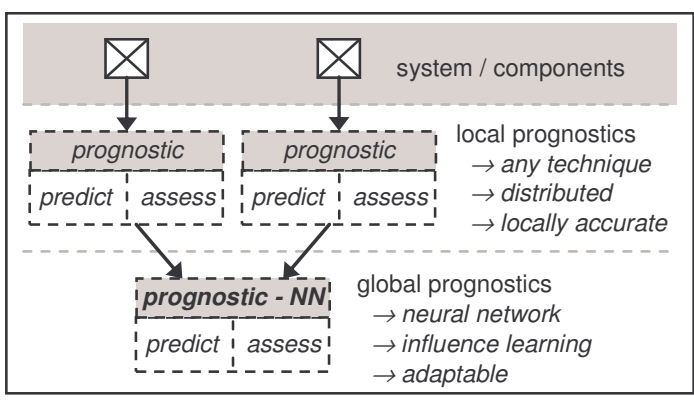

Fig. 5. Schematization of an hybrid and distributed prognostic tool.

Intelligent integrator. As mentioned before, the "global prognostic tool" must handle with the incomplete knowledge of the phenomena and must be adaptable and capable to accommodate with the system evolution. Neural Networks are well fitted for it: they can learn from scenarios and can predict the future evolution of a process. Also, "the transfer function" hasn't to be modeled in legible terms because the model of the system is considered as a black box. In addition, different learning and training algorithms can be chosen (even developed) in concordance with the performances required.

Hybrid aspects. The specific tools used at the local level and the intelligent integrator used at the global one give to the approach proposed an hybrid aspect.

Distributed architecture. The implementation of this framework in an integrated maintenance system, is guided by an "horizontal integrate" policy of the process (Iung., et al., 2001). The supervised parameters, degradation indicators or decision actions are taken as much is possible at the lowest level (the operational level) of the applications. It isn't about a centralized structure like the expert systems, it's more a operational, distributed architecture.

The "weight" of certain parameters with regards to prognostic can be extracted by a permanent survey of the degradations propagation through the process components and by the determination of the degree of influence of those over the final characteristics of the system. The a priori knowledge of the system comportment as well as the online information about its evolution is available for potential users. These are the consequence of system modularity, perfectibility and adaptability.

Finally, note that the integrated approach proposed in this paper can be a good way to incrementally instrument a system: there is no need to directly take the problem on its whole complexity. One can primarily implement several prognostic tools and extend it later.

\section{CONCLUSION AND WORK IN PROGRESS}

Many health monitoring technologies have been developed. They however have traditionally focused on fault detection and isolation within an individual subsystem. 
The researchers in this area are just beginning to address the concepts like prognostics or prognostic integration technologies across subsystems and systems. Hence, the ability to detect and isolate impending faults or to predict the future situation of a system is currently an high priority research topic. In this context, the work reported aims globally at defining a framework for the implantation of a distributed prognostic system to ensure a predictive analysis at all levels of a system.

The main requirements wanted to be meet can be resume as follows:

- identify and assess the degradation degree of components of the analyzed system (local treatments),

- correlate causes with effects from the prediction point of view: first at the local level, and next at the global level of the process,

- learn from scenarios the influence of partial entities on the whole system with neural networks,

- coordinate the local predicted states with the global performance criteria (from local prediction to global prognostic).

The work is still in progress and the developments are at present extended in tree principal ways. First, the definition of prognostic as the association of a prediction and an assessment processes is more precisely studied. Secondly, the application of neural networks as tool for a global prognostic is been investigated. Finally, the implementation of the studied framework is in progress at a French industrial partner for the monitoring of high speed trains motors.

To conclude, let us underline a point of interest of the global work which is not discussed in the paper.

From the industrial point of view, this work is in coherence with actual maintenance trends like "e-maintenance systems" or "intelligent sensors networks" and with the increasing interest for emerging technologies like "web-services".

\section{REFERENCES}

Byington, C., M. Roemer, G. Kacprzynski and T. Galie (2002). Prognostic Enhancements to diagnostic Systems for Improved Conditionbased maintenance. In. 2002 IEEE Aerospace Conference, Big Sky, USA.

Chelidze D., J.P. Cusumano, and A. Charterjee (2002). A Dynamical Systems Approach to Damage Evolution Tracking, part 1: The experimental method. Journal of Vibration and Acoustics, 124, 250-257.

Chiang L.H., E. Russel, and R. Braatz (2001). Fault detection and diagnosis in industrial systems. Springer-Verlag, London.

Ciarapica, F.E. and G. Giacchetta (2006). Managing the condition-based maintenance of a combinedcycle power plant: an approach using soft computing techniques. Journal of Loss Prevention in the Process Industries, 19, 316325.
Dong, Y.L., Y.J. Gu, K. Yang, W.K. Zhang (2004). A combining condition prediction model and its application in power plant. In: Proceedings of 2004 International Conference on Machine Learning and Cybernetics, Shanghai, China, 6, 3474-3478.

EN 13306, june (2001). Maintenance terminology. European Standard, CEN/TC 319 - AFNOR.

Farrar C.R., F. Hemez, G. Park, A.N. Robertson, H. Sohn and T.O. Williams (2003). A Coupled Approach to Developing Damage Prognosis Solutions. In: Damage Assessment of Structures - The 5th International Conference on Damage Assessment of Structures (DAMAS 2003), Southampton, UK.

Freitas, N., I.M. Macleod and J.S. Maltz, (1999). Neural networks for pneumatic actuator fault detection. Transactions of the SAIEE, 90, 28-34.

Iung, B, E. Neunreuther and G. Morel (2001). Engineering Process of Integrated-Distributed Shop Floor Architecture based on Interoperable Field Components. International Journal of Computer Integrated Manufacturing, 14(3), 246262.

Iung B., G. Morel and J.B. Leger (2003). Proactive maintenance strategy for harbour crane operation improuvement. Robotica, 21, 313-324.

Jardine, A.K.S., D. Lin and D. Banjevic (2006). A review on machinery diagnostics and prognostics implementing condition-based maintenance. Mechanical Systems and Signal Processing, 20, 1483-1510.

Leger, J.B. and G. Morel (2001). Integration of maintenance in the enterprise: towards an enterprise modeling based framework compliant with proactive maintenance strategy. Production Planning and Control, 12(2), 176-187.

Lin, D. and V. Makis (2003), Recursive filters for a partially observable system subject to random failure. Advances in Applied Probability, 35, 207-227.

Rytter, A. (1999). Vibration Based Inspection of Civil Engineering Structures. PhD Thesis, Aalborg University, Denmark.

Wang, P. and G. Vachtsevanos (2001). Fault prognostics using dynamic wavelet neural networks. AI EDAM - Artificial Intelligence for Engineering Design Analysis and Manufacturing, 15, 349-365.

Wang, W.Q., M.F. Goldnaraghi and F. Ismail (2004). Prognosis of machine health condition using neuro-fuzzy systems. Mechanical Systems and Signal Processing, 18, 813-831.

Yam, R.C.M., P.W. Tse, L. Li and P. Tu, (2001). Intelligent predictive decision support system for condition-based maintenance, International Journal of Advanced Manufacturing Technology, 17, 383-391

Zhang, G., B.E. Patuwo and M.Y. Hu (1998). Forecasting with artificial neural networks: the state of the art. International Journal of Forecasting, 14, 35-62. 